\title{
On the Dirichlet Problem For Degenerate Elliptic Equations
}

By

\section{J. H. CHABROWSKI*}

\section{\$1. Introduction}

The purpose of this paper is to study the Dirichlet problem with $L^{2}$-boundary data for degenerate elliptic equations of the form (1) (see Section 1). The degeneracy of the ellipticity is controlled by a function $m$ satisfying conditions (2) and (3). Degenerate elliptic equations, with $m$ satisfying (2), have been widely examined by Murthy and Stampacchia [5]. Further extensions of their results can be found in Trudinger [6]. In particular, the Dirichlet problem in the above mentioned papers, was solved in the case when a boundary data is a trace of a function from a suitable Sobolev space. Here we discuss more general situations when a boundary data belongs to $L^{2}$. For uniformly elliptic equations this problem was solved in [1], [2] and [3] (all historical references can be found in [1] and [2]). To solve the Dirichlet problem with $L^{2}$-boundary data we impose on $m$ an additional condition (3), which allows us to recover a boundary function in the sense of $L^{2}$-convergence. Therefore the equation (1) is uniformly elliptic in a neighbourhood of a boundary and degenerates in an interior part of a set.

The plan of this paper is as follows. Section 1 contains some preliminary work. In Section 2 we examine traces of solutions in $H_{l o c}^{1,2}(m, m)$. In particular we obtain a sufficient condition for a solution in $H_{l o c}^{1,2}(m, m)$ to have an $L^{2}$-trace on the boundary (see Theorem 2). This theorem justifies our approach to the Dirichlet problem adopted in this work. In Section 3 we solve the Dirichlet problem for a boundary data in $L^{\infty}$. In the final part of the paper, Section 4, we

Communicated by S. Matsuura, May 22, 1985. Revised September 15, 1986.

*Department of Mathematics, University of Queensland, St. Lucia QLD, Australia. 
solve the Dirichlet problem for a boundary data in $L^{2}$, under more restrictive condition on $m$, namely $m \in L^{\infty}$.

Finally, we point out that the methods of this paper are not new and have appeared in the author's earlier papers [1] and [2].

\section{§ 2. Preliminaries}

Let $Q \subset \boldsymbol{R}_{n}$ be a bounded domain with the boundary $\partial Q$ of class $C^{2}$. In $Q$ we consider the equation of the form

$$
L u=-\sum_{j=1}^{n} D_{j}\left[\sum_{i=1}^{n} a_{i j}(x) D_{i} u+d_{i}(x) u\right]+\sum_{i=1}^{n} b_{i}(x) D_{i} u+c(x) u=f(x),
$$

whose coefficients are assumed to be measurable functions on $Q$.

To formulate further assumptions on the coefficients of $L$ we introduce a non-negative function $m$ on $Q$ such that

$$
m \in L^{s}(Q) \text { and } m^{-1} \in L^{t}(Q) \text { with } \frac{1}{s}+\frac{1}{t} \leq \frac{2}{n} .
$$

We also assume that there exist constants $\beta$ and $\beta_{1}$ and a neighbourhood $N$ of $\partial Q$ such that

$$
0<\beta \leq m(x) \leq \beta_{1}
$$

for all $x \in N$.

We denote a distance from $x \in Q$ to $\partial Q$ by $r(x)$.

Throughout this paper we make the following assumptions on the coefficients of $L$

(A) The operator $L$ is elliptic, that is,

$$
m(x)|\xi|^{2} \leq \sum_{i, j=1}^{n} a_{i j}(x) \xi_{i} \xi_{j}
$$

for all $\xi \in \boldsymbol{R}_{n}$ and almost all $x \in Q \quad$ Moreover we assume that $m^{-1} a_{i j} \in$ $L^{\infty}(Q)$ and that $D_{i} a_{i j}$ exist on $N$ with

$$
D_{i} a_{i j} \cdot r^{\alpha} \in L^{\infty}(N), O<\alpha<1, \quad(i, j=1, \cdots, n) .
$$

(B) $b_{i} m^{-1 / 2}, \quad d_{i} m^{-1 / 2} \in L^{2 \bar{q}}(Q) \quad(i=1, \cdots, n), c \in L^{\bar{q}}(Q)$ with $\bar{q}>q$, where $\mathrm{q}$ is given by

$$
(2 q)^{-1}=2^{-1}-\left(2^{\sharp}\right)^{-1}=n^{-1}-(2 t)^{-1} .
$$

(C) $\mathrm{fm}^{-1}{ }^{2} r^{\theta} \in L^{2}(Q)$, where $2 \leq \theta<3$.

We associate with the function $m$ the weighted Sobolev spaces. The completion of the space $C^{1}(\bar{Q})$ with respect to the norm 


$$
\|u\|_{H^{1,2}(Q, m, m)}=\|u\|_{m, 2}+\|D u\|_{m, 2}
$$

is denoted by $H^{1,2}(Q, m, m) \equiv H^{1,2}(m, m)$. Here $\|\cdot\|_{m, 2}$ denotes the norm in the space $L^{2}(Q, m)$ of all functions $u$ such that

$$
\|u\|_{m, 2}^{2}=\int_{Q}|u(x)|^{2} m(x) d x<\infty .
$$

The closure of the space $C_{0}^{1}(Q)$ in $H^{1,2}(m, m)$ is denoted by $H_{0}^{1,2}(m, m)$.

Throughout this paper we frequently make use of the Sobolev inequality:

there exists a constant $A=A(m, n, t)$ such that

$$
\|u\|_{2^{\sharp}}<A\|D u\|_{m, 2}
$$

for all $u \in H_{0}^{1,2}(m, m)$.

The norm in $H_{0}^{1,2}(m, m)$ is equivalent to $\|D u\|_{m, 2}$, that is, we have

$$
\|D u\|_{m, 2} \leq\|u\|_{H^{1,2}(m, m)} \leq B\|D u\|_{m, 2},
$$

where $B>0$ is a constant independent of $u_{0}$ (For the proofs of (4) and (5) we refer to [5] Theorem 3.2 and Corollaries 3.3 and 3.5).

A function $u$ defined on $Q$ is said to belong to $H_{l o c}^{1,2}(m, m)$ if $\zeta u \in H_{0}^{1,2}(m, m)$ for every $\zeta \in C_{0}^{1}(Q)$.

A function $u(x)$ is said to be a weak solution of the equation (1) if $u \in H_{l o c}^{1,2}(m, m)$ and $u$ satisfies

$$
\int_{Q}\left[\sum_{i, j=1}^{n} a_{i j} D_{i} u D_{j} v+\sum_{i=1}^{n} d_{i} u D_{i} v+\sum_{i=1}^{n} b_{i} D_{i} u v+c u \bullet v\right] d x=\int_{Q} f v d x
$$

for every $v \in H^{1,2}(m, m)$ with compact support in $Q$.

It follows from the regularity of $\partial Q$ that there exists a number $\delta_{0}>0$ such that for $\delta \in\left(0, \delta_{0}\right]$ the domain

$$
Q_{\delta}=Q \cap\left\{x ; \min _{y \in \partial Q}|x-y|>\delta\right\}
$$

with the boundary $\partial Q_{\delta}$, possesses the following property: to each $x_{0} \in \partial Q$ there is a unique point $x_{\delta}\left(x_{0}\right) \in \partial Q_{\delta}$ such that $x_{\delta}\left(x_{0}\right)=x_{0}-\delta \nu\left(x_{0}\right)$, where $\nu\left(x_{0}\right)$ is the outward normal to $\partial Q$ at $x_{0}$. The inverse mapping of $x_{0} \rightarrow x_{\delta}\left(x_{0}\right)$ is given by the formula $x_{0}=x_{\delta}+\delta \nu_{\delta}\left(x_{\delta}\right)$, where $\nu_{\delta}\left(x_{\delta}\right)$ is the outward normal to $\partial Q_{\delta}$ at $x_{\delta}$.

Let $x_{\delta}$ denote an arbitrary point of $\partial Q_{\delta}$. For fixed $\delta \in\left(0, \delta_{0}\right]$ let

$$
\begin{aligned}
& A_{\varepsilon}=\partial Q_{\delta} \cap\left\{x ;\left|x-x_{\delta}\right|<\varepsilon\right\}, \\
& B_{\varepsilon}=\left\{x ; x=\tilde{x}_{\delta}+\delta \nu_{\delta}\left(\tilde{x}_{\delta}\right), \tilde{x}_{\delta} \in A_{\varepsilon}\right\},
\end{aligned}
$$


and

$$
\frac{d S_{\delta}}{d S_{0}}=\lim _{\varepsilon \rightarrow 0} \frac{\left|A_{\varepsilon}\right|}{\left|B_{\varepsilon}\right|}
$$

where $|A|$ denotes the (n-1)-dimensional Hausdorff measure of a set $A$. Mikhailov [4] proved that there exists a positive number $r$ such that

$$
\gamma^{-2} \leq \frac{d S_{\delta}}{d S_{0}} \leq \gamma^{2}
$$

and

$$
\lim _{\delta \rightarrow 0} \frac{d S_{\delta}}{d S_{0}}=1
$$

uniformly with respect to $x_{\delta} \in \partial Q_{\delta}$.

According to Lemma 1 in [3], p. 382, the distance $r(x)$ belongs to $C^{2}\left(\bar{Q}-Q_{\delta_{0}}\right)$, if $\delta_{0}$ is sufficiently small. Denote by $\rho(x)$ the extension of $r$ into $\bar{Q}$ satisfying the following properties: $\rho(x)=r(x)$ for $x \in \bar{Q}-Q_{\delta_{0}}$, $\rho \in C^{2}(Q), \rho(x) \geq \frac{3 \delta_{0}}{4}$ in $Q_{\delta_{0}}, \gamma_{1}^{-1} r(x) \leq \rho(x) \leq \gamma_{1} r(x)$ in $Q$ for some positive constant $\gamma_{1}, \partial Q_{\delta}=\{x ; \rho(x)=\delta\}$ for $\delta \in\left(0, \delta_{0}\right]$ and finally $\partial Q=$ $\{x ; \rho(x)=0\}$. We may also assume that $\bar{Q}-Q_{\delta_{0}} \subset N$.

We will use the surface integrals $M_{1}(\delta)=\int_{\partial Q}\left|u\left(x_{\delta}(x)\right)\right|^{2} d S_{x}$ and $M(\delta)=\int_{\partial Q_{\delta}}|u(x)|^{2} d S_{x}$, where $u \in H_{l o c}^{1,2}(m, m)$ and the values on $\partial Q$ and $\partial Q_{\delta}$, respectively, are understood in the sense of traces (see Theorem 3.9 in [4]).

Note that if $M(\delta)$ is bounded on $\left(0, \delta_{0}\right]$ and

$$
\int_{Q} u(x)^{2} m(x) d x<\infty
$$

then for every $0 \leq \mu<1$ there exists a constant $C>0$ such that

$$
\int_{Q_{\delta}} \frac{u(x)^{2} m(x)}{(\rho(x)-\delta)^{\mu}} d x \leq C
$$

for all $\delta \in\left(0, \delta_{0} / 2\right]$. Indeed, for $\delta \in\left(0, \delta_{0} / 2\right]$ we have

$$
\begin{aligned}
& \int_{Q_{\delta}} \frac{u(x)^{2} m(x)}{(\rho(x)-\delta)^{\mu}} d x \leq \int_{Q_{\delta}-Q_{\delta_{0}}} \frac{u^{2} m}{(\rho-\delta)^{\mu}} d x+\int_{Q_{\delta_{0}}} \frac{u^{2} m}{(\rho-\delta)^{\mu}} d x \\
& \quad \leq \beta_{1} \int_{\delta}^{\delta_{0}}(s-\delta)^{-\mu} \int_{\partial Q} u^{2} d S_{x}+\left(\frac{2}{\delta_{0}}\right)^{\mu} \int_{Q_{\delta}} u^{2} m d x \\
& \quad \leq \beta_{1}\left[\delta_{0}^{1-\mu} \sup _{0<s<\delta_{0}} \int_{\partial Q_{s}} u^{2} d S_{x}+\left(\frac{2}{\delta_{0}}\right)^{\mu} \int_{Q_{\delta_{0}}} u^{2} m d x\right]
\end{aligned}
$$




\section{§3. Properties of $\mathbb{M}$ and $\mathbb{M}_{1}$}

To study $M$ and $M_{1}$ we need the following modification of Lemma 1 in [2].

Lemma 1 . Suppose that $u \in H_{l o c}^{1,2}(m, m)$ and that

$$
\int_{Q}|D u(x)|^{2} m(x) r(x) d x<\infty .
$$

Then if $0 \leq \mu<1$ and $0<\delta_{1} \leq \delta_{0} / 2$ we have for $\delta \in\left(0, \delta_{1} / 2\right]$

$$
\begin{aligned}
& \int_{Q_{\delta}} \frac{u(x)^{2} m(x)}{(\rho(x)-\delta)^{\mu}} d x \leq K\left[\delta_{1}^{-\mu} \int_{Q_{\delta_{1}}} u(x)^{2} m(x) d x\right. \\
& \left.\quad+\delta_{1}^{1-\mu} \int_{\partial Q_{\delta_{1}}} u(x)^{2} d S_{x}+\delta_{1}^{1-\mu} \int_{Q_{\delta_{\delta}-Q_{\delta_{1}}}}|D u(x)|^{2}(\rho(x)-\delta) d x\right],
\end{aligned}
$$

where $K$ is a constant independent of $\delta_{1}$ and $\delta$.

Proof. First we observe that by (3) we have

$$
\int_{Q_{\delta}-Q_{\delta_{1}}}|D u(x)|^{2}(\rho(x)-\delta) d x \leq \beta^{-1} \int_{Q}|D u(x)|^{2} m(x) r(x) d x<\infty .
$$

Let $\delta \in\left(0, \delta_{1} / 2\right]$ and put

$$
\int_{Q_{\delta}} \frac{u^{2} m}{(\rho-\delta)^{\mu}} d x=\int_{Q_{\delta}-Q_{\delta_{1}}} \frac{u^{2} m}{(\rho-\delta)^{\mu}} d x+\int_{Q_{\delta_{1}}} \frac{u^{2} m}{(\rho-\delta)^{\mu}} d x .
$$

Since $\rho(x) \geq \delta_{1}$ on $Q_{\delta_{1}}$ we have

$$
\int_{Q_{\delta_{1}}} \frac{u^{2} m}{(\rho-\delta)^{\mu}} d x \leq\left(\frac{2}{\delta_{1}}\right)^{\mu} \int_{Q_{\delta_{1}}} u^{2} m d x .
$$

Now we note that

$$
\begin{aligned}
& \int_{Q_{\delta}-Q_{\delta_{1}}} \frac{u^{2} m}{(\rho-\delta)^{\mu}} d x \leq \beta_{1} \int_{\delta}^{\delta_{1}}(t-\delta)^{-\mu} \int_{\partial Q} u\left(x_{t}\left(x_{0}\right)\right)^{2} \frac{d S_{t}}{d S_{0}} d S_{0} d t \\
& \leq \beta_{1} \gamma^{2} \int_{\delta}^{\delta_{1}}(t-\delta)^{-\mu} \int_{\partial Q} u\left(x_{t}\left(x_{0}\right)\right)^{2} d S_{0} d t .
\end{aligned}
$$

As $\int_{\partial Q} u\left(x_{t}(x)\right)^{2} d S_{x}$ is absolutely continuous on $\left[\delta, \delta_{1}\right](\delta>0)$, integrating by parts and using Young's inequality we obtain

$$
\int_{Q_{\delta}-Q_{\delta_{1}}} \frac{u^{2} m}{(\rho-\delta)^{\mu}} d x \leq \frac{\beta_{1} \gamma^{2} \delta_{1}^{1-\mu}}{1-\mu} \int_{\vartheta Q} u\left(x_{\delta_{1}}(x)\right)^{2} d S_{x}
$$




$$
\begin{aligned}
& +\frac{2 \gamma^{2} \beta_{1}}{1-\mu} \int_{\delta}^{\delta_{1}}(t-\delta)^{1-\mu} \int_{\partial Q}\left|u\left(x_{t}\left(x_{0}\right)\right)\right|\left|D u\left(x_{t}\left(x_{0}\right)\right)\right|\left|\frac{\partial}{\partial t} x_{t}\left(x_{0}\right)\right| d S_{0} d t \\
& \leq \frac{\beta_{1} \gamma^{4} \delta_{1}^{1-\mu}}{1-\mu} \int_{\partial Q_{\delta_{1}}} u^{2} d S_{x}+\frac{2 \gamma^{4} \beta_{1}}{1-\mu} \int_{Q_{\delta}-Q_{\delta_{1}}}|u(x)||D u(x)|(\rho-\delta)^{1-\mu} d x \\
& \leq \frac{\beta_{1} \gamma^{4} \delta_{1}^{1-\mu}}{1-\mu} \int_{\partial Q_{\delta_{1}}} u^{2} d S_{x}+\frac{2 \gamma^{4} \beta_{1} \varepsilon}{1-\mu} \int_{Q_{\delta}-Q_{\delta_{1}}} \frac{u^{2}}{(\rho-\delta)^{\mu}} d x \\
& +\frac{2 \gamma^{4} \beta_{1} \delta_{1}^{1-\mu}}{\varepsilon(1-\mu)} \int_{Q_{\delta}-Q_{\delta_{1}}}|D u|^{2}(\rho-\delta) d x \text { for all } \varepsilon>0 .
\end{aligned}
$$

Now choosing $\frac{2 \beta_{1} \gamma^{4} \varepsilon}{1-\mu}=\frac{1}{2}$ the result follows.

Lemma 2. Let $u \in H_{l o c}^{1,2}(Q)$ be a solution of (1) and let $0<\delta_{1}<\delta_{2}<\delta_{0}$. Then

$$
\int_{Q_{\delta_{2}}}|D u(x)|^{2} m(x) d x \leq C\left[\int_{Q_{\delta_{1}}} u(x)^{2} m(x) d x+\int_{Q_{\delta_{1}}} f(x)^{2} m(x)^{-1} d x\right],
$$

where $C>0$ is a constant depending on the norms of the coefficients of $L$, $\delta_{1}$ and $\delta_{2}$.

Proof. We commence with the following observation. Let $\Omega$ be a relatively compact subset of $Q$ such that

$$
\left\|\sum_{i=1}^{n} b_{i} m^{-1 / 2}\right\|_{L^{2 q}(\Omega)}, \| \sum_{i=1}^{n} d_{i} m^{-1 / 2}||_{L^{2 q}(\Omega)} \text { and }\|c\|_{L^{2}(\Omega)}
$$

are sufficiently small then

$$
\int_{\Omega}|D u|^{2} \zeta^{2} m d x \leq \mathrm{Const}\left[\int_{\Omega} u^{2}\left(\zeta^{2}+|D \zeta|^{2}\right) m d x+\int_{\Omega} f^{2} \zeta^{2} m^{-1}\right]
$$

for every $C^{1}$-function $\zeta$ with compact support in $\Omega$ (see Lemma 8.5 in [5]). The assertion then follows from the compactness of $\bar{Q}_{\delta_{1}}$.

The following result is crucial in the subsequent treatment of the Dirichlet problem.

Theorem 1. Let $u$ be a solution of (1) belonging to $H_{l o c}^{1,2}(m, m)$, then the following conditions are equivalent

(I) $\quad M(\delta)$ is a bounded function on $\left(0, \delta_{0}\right]$,

(II) $\int_{Q}|D u(x)|^{2} m(x) r(x) d x<\infty$,

(III) There exists $\lim _{\delta \rightarrow 0+} M_{1}(\delta)<\infty$. 
Proof. Put

$$
v(x)= \begin{cases}u(x)(\rho(x)-\delta) & \text { for } x \in Q_{\delta} \\ 0 & \text { for } x \in Q-Q_{\delta}\end{cases}
$$

for $0<\delta<\frac{\delta_{0}}{4}$. It is clear that $v$ is a legitimate test function in (6) and on substitution we obtain

$$
\begin{aligned}
& \int_{Q_{\delta}} \sum_{i, j=1}^{n} a_{i j} D_{i} u D_{j} u(\rho-\delta) d x+\int_{Q_{\delta}} \sum_{i, j=1}^{n} a_{i j} D_{i} u \circ u D_{j} \rho d x \\
& \quad+\int_{Q_{\delta}} \sum_{i=1}^{n}\left(d_{i}+b_{i}\right) D_{i} u \circ u(\rho-\delta) d x+\int_{Q_{\delta}} \sum_{i=1}^{n} d_{i} u^{2} D_{i} \rho d x+\int_{Q_{\delta}} c u^{2}(\rho-\delta) d x \\
& \quad=\int_{Q_{\delta}} f u(\rho-\delta) d x .
\end{aligned}
$$

The proof is similar to that of Theorem 1 in [2], but in our situation more care is needed to estimate the resulting integrals in (10).

The proof of "I $\Rightarrow I I$ ". Let us denote the integrals on the left side of (10) by $J_{1}, \cdots, J_{5}$ By virtue of (A) we have

$$
J_{1} \geq \int_{Q_{\delta}}|D u|^{2}(\rho-\delta) m d x
$$

To estimate $J_{2}$ we set

$$
\begin{aligned}
J_{2} & =\int_{Q_{\delta}} \sum_{i, j=1}^{n} a_{i j} D_{i} u \cdot u D_{j} \rho \Phi d x+\int_{Q_{\delta}} \sum_{i, j=1}^{n} a_{i j} D_{i} u \cdot u D_{j} \rho(1-\Phi) d x \\
& =J_{2}^{\prime}+J_{2}^{\prime \prime},
\end{aligned}
$$

where $\Phi$ is a smooth function on $\bar{Q}$ such that $\Phi=1$ on $Q-Q_{\delta_{0} / 2}, \Phi=0$ on $Q_{\delta_{0}}$ and $0 \leq \Phi \leq 1$ on $\bar{Q}$.

Since $0<\delta<\delta_{0} / 4$, by Green's formula we have

$$
\begin{aligned}
J_{2}^{\prime} & =\frac{1}{2} \int_{Q_{\delta}} \sum_{i, j=1}^{n} a_{i j} D_{i}\left(u^{2}\right) D_{j} \rho \Phi d x \\
& =-\frac{1}{2} \int_{\partial Q_{\delta}} \sum_{i, j=1}^{n} a_{i j} D_{i} \rho D_{j} \rho u^{2} d S_{x}-\frac{1}{2} \int_{Q_{\delta}} \sum_{i, j=1}^{n} D_{i}\left(a_{i j} D_{j} \rho \Phi\right) u^{2} d x .
\end{aligned}
$$

On the other hand supp $(1-\Phi) \subset Q_{\delta_{0} / 2}$, therefore applying Young's inequality and Lemma 2 to $J_{2}^{\prime \prime}$ we arrive at the following estimate for $J_{2}$

$$
\left|J_{2}\right| \leq \frac{1}{2} \int_{\partial Q_{\delta}} \sum_{i, j=1}^{n} a_{i j} D_{i} \rho D_{j} \rho u^{2} d S_{x}+C_{1} \int_{Q_{\delta}} u^{2} m\left(1+\rho^{-\alpha}\right) d x
$$


where a constant $G_{1}>0$ depends on $\left\|a_{i j} m^{-1}\right\|_{L^{\infty}}$ and $\left\|D_{i} a_{i j} m^{-1} \rho^{\alpha}\right\|_{L^{\infty}}$. To estimate $J_{3}$ we use a decomposition

$$
\begin{aligned}
J_{3} & =\int_{Q_{\delta_{0}}} \sum_{i=1}^{n}\left(d_{i}+b_{i}\right) D_{i} u \cdot u(\rho-\delta) d x+\int_{Q_{\delta}-Q_{\delta_{0}}} \sum_{i=1}^{n}\left(d_{i}+b_{i}\right) D_{i} u \cdot u(\rho-\delta) d x \\
& =J_{3}^{\prime}+J_{3}^{\prime \prime}
\end{aligned}
$$

Since $L^{2 \bar{q}} \subset L^{2 q}$, using Hölder's inequality, Theorem 3.1 from [5] and Lemma 2 we get

$$
\begin{aligned}
\left|J_{3}^{\prime}\right| & \leq\left\|\sum_{i=1}^{n}\left(b_{i}+d_{i}\right)\right\|_{L^{2 q}\left(Q_{\delta_{0}}\right)}\left\|D u(\rho-\delta)^{1 / 2} m^{1 / 2}\right\|_{L^{2}\left(Q_{\delta_{0}}\right)}\|u(\rho-\delta)\|_{L^{2^{\#}\left(Q_{\delta_{0}}\right)}} \\
& \leq C_{2}\left[\int_{Q} f^{2} m^{-1} \rho^{\theta} d x+\int_{Q_{\delta_{0} / 2}} u^{2} m d x\right]
\end{aligned}
$$

where $C_{2}>0$ depends on $\left\|\sum_{i=1}^{n}\left(\mathrm{~b}_{i}+d_{i}\right) m^{-1 / 2}\right\|_{L^{2 \bar{q}}}$ and a constant from Lemma 2. To estimate $J_{3}^{\prime \prime}$ we first use the Hölder inequality to obtain

$$
\begin{aligned}
\left|J_{3}^{\prime \prime}\right| & \leq\left\|\sum_{i=1}^{n}\left(b_{i}+d_{i}\right) m^{-1 / 2}\right\|_{L^{2 \bar{q}}\left(O_{\delta}-O_{\delta_{0}}\right)}\left\|(\rho-\delta)^{-\varepsilon}\right\|_{L^{s_{1}}\left(Q_{\delta}-Q_{\delta_{0}}\right)} \\
& \times\left\|u(\rho-\delta)^{1 / 2+\varepsilon / 2}\right\|_{L^{2^{\sharp}}\left(Q_{\delta}-Q_{\delta_{0}}\right)}\left\|D u(\rho-\delta)^{1 / 2+\varepsilon / 2} m^{1 / 2}\right\|_{L^{2}\left(Q_{\delta}-Q_{\delta_{0}}\right)},
\end{aligned}
$$

with $\frac{1}{2 \bar{q}}+\frac{1}{\mathrm{~s}_{1}}=\frac{1}{2 \mathrm{q}}, \frac{1}{2 \mathrm{q}}=\frac{1}{2}-\frac{1}{2^{\ddagger}}, 0<\varepsilon<\frac{1}{\mathrm{~s}_{1}}$.

Hence be the Sobolev inequality (4) we have

$$
\begin{aligned}
& \left\|\left.u(\rho-\delta)^{1 / 2+\varepsilon / 2}\right|_{L^{2^{\sharp}}\left(Q_{\delta}-Q_{\delta_{0}}\right)} \leq\right\| u(\rho-\delta)^{1 / 2+\varepsilon / 2} \|_{L^{2^{\sharp}\left(Q_{\delta}\right)}} \\
& \quad \leq A\left[\left\|\left.D u(\rho-\delta)^{1 / 2} m^{1 / 2}\right|_{L^{2}\left(Q_{\delta^{\delta}}\right)}+\right\| u(\rho-\delta)^{\varepsilon / 2-1 / 2} m^{1 / 2} \|_{L^{2}\left(Q_{\delta}\right)}\right],
\end{aligned}
$$

so that

$$
\left|J_{3}^{\prime \prime}\right| \leq \delta_{0}^{-\varepsilon+1 / s_{1}} \int_{Q_{\delta}}|D u|^{2} m \rho d x+C_{3} \int_{Q_{\delta}} u^{2} m(\rho-\delta)^{\varepsilon-1} d x,
$$

where $C_{3}>0$ depends on the norms of $d_{i}+b_{i}, A, q$ and $s_{1}$.

Similarly we write

$$
\begin{aligned}
\left|J_{4}\right| & \leq \sup _{Q}|D \rho|\left[\int_{Q_{\delta_{0}}} \sum_{i=1}^{n}\left|d_{i}\right| u^{2} d x+\int_{Q_{\delta}-Q_{\delta_{0}}} \sum_{i=1}^{n}\left|d_{i}\right| u^{2} d x\right] \\
& =\sup _{Q}|D \rho|\left[J_{4}^{\prime}+J_{4}^{\prime \prime}\right] .
\end{aligned}
$$

It follows then from the Hölder and Sobolev inequalities that 


$$
\begin{aligned}
\left|J_{4}^{\prime}\right| & \leq\left\|\sum_{i=1}^{n} d_{i} m^{-1 / 2}\right\|_{L^{2 q}\left(Q_{\delta_{0}}\right)} \cdot\left\|u m^{1 / 2}\right\|_{L^{2}\left(Q_{\delta_{0}}\right)} \cdot\|u\|_{L^{2^{\#}}\left(Q_{\delta_{0}}\right)} \\
& \leq A\left\|\sum_{i=1}^{n} d_{i} m^{-1 / 2}\right\|_{L^{2 q}\left(Q_{\delta_{0}}\right)}\left\|u m^{1 / 2}\right\|_{L^{2}\left(Q_{\delta_{0}}\right)}\left[\left\|u m^{1 / 2}\right\|_{L^{2}\left(Q_{\delta_{0}}\right)}+\left\|D u \cdot m^{1 / 2}\right\|_{L^{2}\left(Q_{\delta_{0}}{ }^{2}\right.}\right] .
\end{aligned}
$$

Consequently applying Lemma 2 we arrive at the estimate

$$
\left|J_{4}^{\prime}\right| \leq C_{4}\left[\int_{Q} \mathrm{f}^{2} m^{-1} \rho^{\theta} d x+\int_{Q_{\delta_{0} / 2}} u^{2} m d x\right]
$$

where $C_{4}$ is a constant depending on the norm of $\sum_{i=1}^{n}\left|d_{i}\right|, A$ and a constant from Lemma 2. To estimate $J_{4}^{\prime \prime}$ we first observe that by (3) we have

$$
\left\|\sum_{i=1}^{n} d_{i}\right\|_{L^{2 \bar{q}}\left(Q_{\delta}-Q_{\left.\delta_{0}\right)}\right.} \leq \beta_{1} \|\left.\sum_{i=1}^{n} d_{i} m\right|_{L^{2 \bar{q}}(Q)}<\infty
$$

for $\delta \in\left(0, \delta_{0}\right]$. Then using the Hölder inequality, we obtain

$$
\begin{aligned}
\left|J_{4}^{\prime \prime}\right| & \leq\left[\int_{Q_{\delta}-Q_{\delta_{0}}}\left(\sum_{i=1}^{n}\left|d_{i}\right|\right)^{2 \bar{q}} d x\right]^{1 / 2 \bar{q}}\left[\int_{Q_{\delta}-Q_{\delta_{0}}}(\rho-\delta)^{-\varepsilon_{1} s_{1}} d x\right]^{1 / s_{1}} \\
& \times\left[\int_{Q_{\delta}-Q_{\delta_{0}}} u^{2}(\rho-\delta)^{-\varepsilon_{2}} d x\right]^{1 / 2}\left[\int_{Q_{\delta}-Q_{\delta_{0}}}\left|u(\rho-\delta)^{\varepsilon_{1}+\varepsilon_{2} / 2}\right|^{2^{\sharp}} d x\right]^{1 / 2^{\#}}
\end{aligned}
$$

with $\frac{1}{2 \bar{q}}+\frac{1}{s_{1}}=\frac{1}{2 q}, \frac{1}{2^{\#}}+\frac{1}{2 q}+\frac{1}{2}=1,0<\varepsilon<\frac{1}{s_{1}}, \frac{1}{2}<\varepsilon_{1}+\frac{\varepsilon_{2}}{2}$ and $0<$ $\varepsilon_{2}<1$. Let us now apply the Sobolev inequality (4) to obtain

$$
\begin{aligned}
\left|J_{4}^{\prime \prime}\right| \leq \delta_{0}^{1 / s_{1}-\varepsilon_{1}} \int_{Q_{\delta}}|D u|^{2}(\rho-\delta) d x & +C_{5} \int_{Q_{\delta}} u^{2}\left[(\rho-\delta)^{-\varepsilon_{2}}\right. \\
& \left.+(\rho-\delta)^{2 \varepsilon_{1}+\varepsilon_{2}-2}\right] d x
\end{aligned}
$$

where $C_{5}$ is a constant depending on the norm of $\sum_{i=1}^{n} d_{i}, A$ and $s_{1}$. Similarly,

$$
\left|J_{5}\right| \leq \int_{Q_{\delta_{0}}}|c| u^{2}(\rho-\delta) d x+\int_{Q_{\delta}-Q_{\delta_{0}}}|c| u^{2}(\rho-\delta) d x=J_{5}^{\prime}+J_{5}^{\prime \prime}
$$

Now by the Sobolev and Hölder inequalities and Lemma 2 we get

$$
\left|J_{5}^{\prime}\right| \leq C_{6}\left[\int_{Q_{\delta_{0} / 2}} u^{2} m d x+\int_{Q_{\delta_{0}}} f^{2} m^{-1} \rho^{\theta} d x\right]
$$

where $C_{6}>0$ is a constant. On the other hand we have 


$$
\begin{aligned}
\left|J_{5}^{\prime \prime}\right| & \leq\|c\|_{L^{\bar{q}}\left(Q_{\delta}-Q_{\delta_{0}}\right)}\left\|(\rho-\delta)^{-\varepsilon_{3}}\right\|_{L^{s_{1}}\left(Q_{\delta}-Q_{\delta_{0}}\right)}\left\|u(\rho-\delta)^{1 / 2+\varepsilon_{3} / 2}\right\|_{L^{2^{\#}}\left(Q_{\delta}-Q_{\delta_{0}}\right)} \\
& \times\left\|u(\rho-\delta)^{1 / 2+\varepsilon_{3} / 2}\right\|_{L^{2^{\#}}\left(Q_{\delta}-Q_{\delta_{0}}\right)^{\prime}}
\end{aligned}
$$

with $\frac{1}{2 \bar{q}}+\frac{1}{2^{\sharp}}+\frac{1}{s_{1}}+\frac{1}{2}=1,0<\varepsilon_{3}<\frac{1}{s_{1}}$, so that

$$
\left|J_{5}^{\prime \prime}\right| \leq \delta^{1 / s_{1}-\varepsilon_{3}} \int_{Q_{\delta}}|D u|^{2}(\rho-\delta) m d x+C_{7} \int_{Q_{\delta}} u^{2} m(\rho-\delta)^{\varepsilon_{3}-1} d x
$$

where $C_{7}$ depends on $\|c\|_{L^{\bar{q}}}, s_{1}$ and $A$. Finally,

$$
\left|\int_{Q_{\delta}} f \cdot u(\rho-\delta) d x\right| \leq \int_{Q_{\delta}} f^{2} m^{-1}(\rho-\delta)^{\theta} d x+\int_{Q_{\delta}} u^{2} m(\rho-\delta)^{2-\theta} d x .
$$

Inserting the estimates (11) - (19) into (10) we obtain, assuming that $\delta_{0}$ is sufficiently small, that

$$
\begin{aligned}
& \int_{Q_{\delta}}|D u|^{2}(\rho-\delta) m d x \leq C_{8}\left[\int_{Q^{2}} f^{2} m^{-1} \rho^{\theta} d x\right. \\
& \left.\quad+\int_{Q_{\delta}} u^{2} m(\rho-\delta)^{-\mu} d x+\int_{Q_{\delta_{0}} / 2} u^{2} m d x+\sup _{0<\delta<\delta_{0}} \int_{\partial Q_{\delta}} u^{2} d S_{x}\right]
\end{aligned}
$$

for all $\delta \in\left(0, \delta_{0} / 4\right]$, where $\mu=\max \left(\alpha, \theta-2, \varepsilon_{2}, \varepsilon_{3}, 2-\varepsilon_{2}-2 \varepsilon_{1}\right)$ and $C_{8}>0$ depends on $\delta_{0}$ and constants $C_{1}-C_{7}$. Now note that if (I) holds then we have the estimate (9), therefore the condition (II) follows from the monotone convergence theorem.

Proof of $I I \Rightarrow I I I$. From the first part of the proof we deduce that

$$
\begin{aligned}
& \frac{1}{2} \int_{\partial Q_{\delta}} \sum_{i, j=1}^{n} a_{i j} D_{i} \rho D_{j} \rho u^{2} d S_{x}=\int_{Q_{\delta}} \sum_{i, j=1}^{n} a_{i j} D_{i} u D_{j} u(\rho-\delta) d x \\
& \quad-\frac{1}{2} \int_{Q_{\delta}} \sum_{i, j=1}^{n} D_{i}\left(a_{i j} D_{j} \rho\right) u^{2} d x+\int_{Q_{\delta}} \sum_{i=1}^{n}\left(b_{i}+d_{i}\right) D_{i} u \cdot u(\rho-\delta) d x \\
& \quad+\int_{Q_{\delta}} \sum_{i=1}^{n} d_{i} u^{2} D_{i} \rho d x+\int_{Q_{\delta}} c u^{2}(\rho-\delta) d x-\int_{Q_{\delta}} f u(\rho-\delta) d x .
\end{aligned}
$$

It follows from Lemma 1 that

$$
\int_{Q_{\delta}} \frac{u(x)^{2} m(x)}{(\rho(x)-\delta)^{\mu}} d x \leq C, 0 \leq \mu<1
$$

for $\delta \in\left(0, \delta_{0} / 2\right]$, where $G>0$ is independent of $\delta$. 
Repeating the argument from " $I \Rightarrow I I$ " it is easy to show that all integrals are convergent as $\delta \rightarrow 0$. Hence "II $\Rightarrow I^{\prime \prime}$ " follows from (8) and the relationship

$$
M(\delta)-M_{1}(\delta)=\int_{\partial Q} u\left(x_{\delta}(x)\right)^{2}\left[\frac{d S_{\delta}}{d S_{0}}-1\right] d S_{0}
$$

Finally "III $\Rightarrow I$ " follows from the proof "II $\Rightarrow$ III.

\section{§4. Traces in $L^{2}(\partial Q)$ and the Dirichlet Problem}

In this section we first establish the existence of a trace of $u$ on $\partial Q$ in $L^{2}(\partial Q)$, that is, $u\left(x_{\delta}\right)$ converges in $L^{2}(\partial Q)$ as $\delta \rightarrow 0$.

Theorem 2. Let $u$ be a solution of (1) in $H_{l o c}^{1,2}(\mathrm{~m}, \mathrm{~m})$. If one of the conditions I, II or III holds, then there exists a function $\zeta$ belonging to $L^{2}(\partial Q)$ such that $u\left(x_{\delta}\right)$ converges to $\zeta$ in $L^{2}(\partial Q)$.

The proof is identical to the proof of Theorem 4 in [2], therefore we only give an outline. It is obvious that there exists a sequence $\delta_{\nu} \rightarrow 0$ as $\nu \rightarrow \infty$ and a function $\zeta \in L^{2}(\partial Q)$ such that

$$
\lim _{\nu \rightarrow \infty} \int_{\partial Q} u\left(x_{\delta_{\nu}}(x)\right) g(x) d S_{x}=\int_{\partial Q} \zeta(x) g(x) d S_{x}
$$

for each $g \in L^{2}(\partial Q)$. Repeating the argument of Theorem 3 in [2] one can easily prove that the above relation holds true if the sequence $\delta_{\nu}$ is replaced by $\delta$. Finally to prove that $u\left(x_{\delta}\right) \rightarrow \zeta$ in $L^{2}(\partial Q)$ we show that $\lim _{\delta \rightarrow 0} \int_{\partial Q} u\left(x_{\delta}(x)\right)^{2} d S_{x}=\int_{\partial Q} \zeta(x)^{2} d S_{x}$ and the result then follows by uniform convexity of $L^{2}(\partial Q)$.

Theorem 2 justifies the following approach to the Dirichlet problem.

Let $\phi \in L^{2}(\partial Q)$. A weak solution $u$ in $H_{l o c}^{1,2}(\mathrm{~m}, \mathrm{~m})$ of $(1)$ is a solution of the Dirichlet problem with the boundary condition

$$
u(x)=\phi(x) \text { on } \partial Q
$$

if $\lim _{\delta \rightarrow 0} \int_{\partial Q}\left[u\left(x_{\delta}(x)\right)-\phi(x)\right]^{2} d S_{x}=0$.

To establish the existence of a solution of the Dirichlet problem (1)-(22) we need some additional assumptions that will be used only in this section.

(a) There exists a constant $a>-\infty$ such that 


$$
-\sum_{j=1}^{n} D_{j} d_{j}+c \geq a
$$

in the sense of distribution.

(b)

$$
n A_{0}\left[\sum_{i=1}^{n}\left\|b_{i} m^{-1 / 2}\right\|_{2 q}+\sum_{i=1}^{n}\left\|d_{i} m^{-1 / 2}\right\|_{2 q}\right]+A_{0}^{2}\|c\|_{q}<1
$$

with $A_{0}=A \cdot B$, where $A$ and $B$ are constants from the inequalities (4) and (5).

Finally, let us introduce the Hilbert space $\tilde{H}^{1,2}(m, m)$ of all functions $u$ in $H_{l o c}^{1,2}(m, m)$ such that

$$
\|u\|_{H^{1,2}(m, m)}^{2}=\int_{Q} u(x)^{2} m(x) d x+\int_{Q}|D u(x)|^{2} m(x) r(x) d x<\infty .
$$

It is obvious that for all $d<\delta_{0}$

$$
\begin{aligned}
& \|u\|_{H^{1,2}(m, m)}^{2} \leq \max \left(1, \beta_{1} d\right)\left[\int_{Q} \mid D u(x)^{2} r(x) m(x) d x\right. \\
& \left.\quad+\int_{Q} u(x)^{2} m(x) d x+\sup _{0<\delta<d} M(\delta)\right] .
\end{aligned}
$$

We can now state the following existence result.

Theorem 3. Let $\phi \in L^{\infty}(\partial Q)$ and suppose that $\int_{Q}|f|{ }^{p} m^{-1} d x<\infty$ for $p>\frac{n(t-1)}{t-n}$. Then, if the coefficients of $L$ satisfy $(a)$ and $(b)$, the Dirichlet problem (1), (22) has a solution in $H_{l o c}^{1,2}(m, m)$.

Proof. Let $\left\{\phi_{\nu}\right\}$ be a sequence of functions in $C^{1}(\partial Q)$ converging in $L^{2}(\partial Q)$ to $\phi$. In virtue of assumption (b) and Theorem 4.7 in [5] for every $\nu$ there exists a unique solution $u_{\nu} \in H^{1,2}(m, m)$ of the Dirichlet problem

$$
\begin{array}{lll}
L u=f & \text { on } & Q, \\
u=\phi_{\nu} & \text { on } & \partial Q .
\end{array}
$$

Since $\phi \in L^{\infty}(\partial Q)$ we may assume that $\sup _{\nu \geq 1}\left\|\phi_{\nu}\right\|_{\infty}<\infty$. The assumption (b) implies that the maximum principle holds (see Corollary 7.4 in [5]), consequently

$$
\left|u_{\nu}(x)\right| \leq \sup _{\nu \geq 1}|| \phi_{\nu} \|_{\infty}+\int_{Q}|f|{ }^{p} m^{-1} d x
$$

for all $\nu$. Inspection of the proof of Theorem 1 shows that there exists a constant $C>0$ such that 


$$
\begin{aligned}
& \int_{Q}\left|D u_{\nu}\right|^{2} m r d x+\int_{Q} u_{\nu}^{2} m d x+\sup _{0<\delta<d} \int_{\partial Q_{\delta}} u_{\nu}^{2} d x \\
& \leq C\left[\int_{\partial Q} \phi_{\nu}^{2} d S_{x}+\int_{Q_{\delta_{0} / 2}} u_{\nu}^{2} d x+\int_{Q} f^{2} m^{-1} d x\right] .
\end{aligned}
$$

It is clear from (23) and (24) that there exists a subsequence $\left\{u_{\nu_{s}}\right\}$ weakly convergent in $\tilde{H}^{1,2}(m, m)$ to a solution of the Dirichlet problem (1) $-(22)$.

\section{§5. Case $m \in \mathbb{H}^{\infty}(\mathbb{Q})$}

Throughout this section we assume additionally that $m \in L^{\infty}(Q)$. This assumption allows us to consider the boundary data in $L^{2}(\partial Q)$. The right Sobolev space in this situation is $H^{1,2}(1, m)$ which is the completion of $C^{1}(\bar{Q})$ with respect to the norm

$$
\|u\|_{H^{1,2}(Q, 1, m)}^{2}=\|u\|_{L^{2}(Q)}^{2}+\|\left. D u m^{1 / 2}\right|_{L^{2}(Q)} ^{2} \text {. }
$$

We briefly denote this space by $H^{1,2}(m)$. We define spaces $H_{0}^{1,2}(m)$ and $H_{l o c}^{1,2}(m)$ in an obvious way.

We commence with the extensions of Theorems 1 and 2.

Theorem 4. Let $u$ be a solution of (1) belonging to $H_{l o c}^{1,2}(m)$, then the conditions $(I),(I I)$ and $(I I I)$ are equiva'ent.

Theorem 5. Let $u$ be a solution of (1) in $H_{l o c}^{1,2}(m)$. If one of the conditions $(I),(I I)$ or $(I I I)$ holds, then there exists a function $\zeta \in L^{2}(\partial Q)$ such that $u\left(x_{\delta}\right)$ converges to $\zeta$ in $L^{2}(\partial Q)$.

The proofs of these results are essentially the same as of Theorems 1 and 2 and therefore are omitted. We only point out here that the inequality from Lemma 1 , which is crucial in the step $I I \rightarrow I I$, can be stated in the following form: if $u \in H_{l o c}^{1,2}(m), 0 \leq \mu<1$ and $0<$ $\delta_{1} \leq \delta_{0} / 2$ then we have

$$
\begin{aligned}
& \int_{Q_{\delta}} \frac{u(x)^{2}}{(\rho(x)-\delta)^{\mu}} d x \leq K\left[\delta_{1}^{-\mu} \int_{Q_{\delta_{1}}} u(x)^{2} d x+\delta_{1}^{1-\mu} \int_{\partial Q_{\delta_{1}}} u(x)^{2} d S_{x}\right. \\
& \left.\quad+\delta_{1}^{1-\mu} \int_{Q_{\delta}-Q_{\delta_{1}}}|D u(x)|^{2}(\rho(x)-\delta) d x\right]
\end{aligned}
$$


for all $\delta \in\left(0, \delta_{1} / 2\right]$, where $K>0$ is a constant independent of $\delta_{1}$ and $\delta$.

To proceed further let us introduce the equation

$$
L u+\lambda u=f \text {, }
$$

where $\lambda$ is a real parameter.

We establish the following energy estimate

Theorem 6. Let $u \in H_{l o c}^{1,2}(m)$ be a solution of the Dirichlet problem $\left(1_{\lambda}\right)-(22)$. Then there exist positive constants $d, \lambda_{0}$ and $C$ independent of $u$ such that

$$
\begin{gathered}
\int_{Q}|D u(x)|^{2} m(x) r(x) d x+\sup _{0<\delta<d} M(\delta)+\lambda \int_{Q_{g}} u(x)^{2} r(x) d x \\
\leq\left[\int_{\partial Q} \phi(x)^{2} d S_{x}+\int_{Q} f(x)^{2} r(x)^{\theta} m(x)^{-1} d x\right]
\end{gathered}
$$

for $\lambda \geq \lambda_{0}$.

Proof. The proof is similar to that of Theorem 5 in [2] (see also Lemma 1 in [1]). An examination of the proof of Theorem 1 shows that we can write the following estimate

$$
\begin{aligned}
& \int_{Q_{\delta}}|D u|^{2} m(\rho-\delta) d x+\lambda \int_{Q_{\delta}} u^{2}(\rho-\delta) d x \leq G_{1}\left[\int_{Q_{\delta}} f^{2}(\rho-\delta)^{\theta} m^{-1} d x\right. \\
& \left.\quad+\int_{\partial Q_{\delta}} u^{2} d S_{x}+\int_{Q_{\delta}} u^{2}(\rho-\delta)^{-\mu} d x\right]
\end{aligned}
$$

where $\mu=\max \left(\alpha, \theta-2, \varepsilon_{2}, \varepsilon_{3}, 2-\varepsilon_{2}-2 \varepsilon_{1}\right), C_{1}>0$ is a positive constant independent of $u, \lambda$ and $\delta \in\left(0, \delta_{0} / 2\right], \varepsilon_{1}, \varepsilon_{2}$ and $\varepsilon_{3}$ are positive constants introduced in Theorem 1. On the other hand, using (21) we easily arrive at the following estimate

$$
\begin{aligned}
& \sup _{0<\delta<d} \int_{\partial Q_{\delta}} u(x)^{2} d S_{x} \leq C_{2}\left[\int_{Q}|D u|^{2} m \rho d x\right. \\
& \left.\quad+\lambda \int_{Q} u^{2} \rho d x+\int_{Q} f^{2} \rho^{\theta} m^{-1} d x+\int_{Q} u^{2} \rho^{-\mu} d x\right],
\end{aligned}
$$

where $C_{2}>0$ is a constant independent of $u, \lambda$ and $\delta \in\left(0, \delta_{0} / 2\right]$. Letting $\delta \rightarrow 0$ we deduce from (26) that

$$
\int_{Q}|D u|^{2} m \rho d x+\lambda \int_{Q} u^{2} \rho d x \leq C_{1}\left[\int_{\partial Q} \phi^{2} d S_{x}+\int_{Q} f^{2} \rho^{\theta} m^{-1} d x+\int_{Q} u^{2} \rho^{-\mu} d x\right] .
$$

Combining this inequality with (27), we obtain 


$$
\begin{aligned}
& \int_{Q}|D u|^{2} m \rho d x+\lambda \int_{Q} u^{2} \rho d x+\sup _{0<\delta<d} \int_{\partial Q_{\delta}} u^{2} d S_{x} \\
& \leq C_{3}\left[\int_{\partial Q} \phi^{2} d S_{x}+\int_{Q} f^{2} \rho^{\theta} m^{-1} d x+\int_{Q}^{\prime} u^{2} \rho^{-\mu} d x\right]
\end{aligned}
$$

where $C_{3}>0$ is a constant. Now

$$
\int_{Q} u^{2} \rho^{-\mu} d x \leq \frac{d^{1-\mu}}{1-\mu} \sup _{0<\delta \leq d} \int_{\partial Q_{\delta}} u^{2} d S_{x}+\frac{1}{m_{d}^{1+\mu}} \int_{Q} u^{2} \rho d x
$$

for all $0 \leq \mu<1$, with $m_{d}=\inf _{Q_{d}} \rho(x)$.

Taking $\lambda_{0}$ sufficiently large and $d$ sufficiently small the result follows from (28).

Now we are in a position to state the existence result.

Theorem 7. Suppose $\phi \in L^{2}(\partial Q)$. Then there exists a unique solution of the Dirichlet problem $\left(1_{\lambda}\right)-(22)$ for $\lambda \geq \lambda_{0}$.

The proof is identical to that of Theorem 6 in [2].

Theorem 8. Suppose that $\int_{Q} f^{2} m^{-1} d x<\infty$ and let $\phi \in L^{2}(\partial Q)$. If there is a function $\phi_{1} \in H^{1,2}(m)$ such that $\phi_{1}=\phi$ on $\partial Q$ in the sense of trace, then a solution $u \in H_{l o c}^{1,2}(m)$ of the Dirichlet problem $\left(1_{\lambda}\right)-(22)\left(\lambda \geq \lambda_{0}\right)$ is a solution in $H^{1,2}(m)$ of the same problem.

This follows from the fact that any solution of $\left(1_{\lambda}\right)-(22)$ in $H^{1,2}(m)$ is also a solution of the same problem in $H_{l o c}^{1,2}(m)$ and both problems have a unique solution in respective spaces.

\section{§6. Final Remark}

One can also establish the existence of a solution of the Dirichlet problem with $\phi \in L^{2}(\partial Q)$ and with $m$ satisfying (2) and (3) but for the equation

$$
(1 m, \lambda) \quad L u+\lambda m u=f \quad \text { on } Q \text {. }
$$

Indeed, an examination of the proof of Theorem 6 shows that there exist positive constants $\lambda_{0}, d$ and $C$ such that 


$$
\begin{aligned}
& \int_{Q}|D u(x)|^{2} r(x) m(x) d x+\lambda \int_{Q} u(x)^{2} m(x) r(x) d x+\sup _{0<\delta<d} M(\delta) \\
& \quad \leq C\left[\int_{\partial Q} \phi(x)^{2} d S_{x}+\int_{Q} f(x)^{2} r(x)^{\theta} m(x) d x\right]
\end{aligned}
$$

for every solution $u \in H_{l o c}^{1,2}(m, m)$ of $(1 m, \lambda)-(22)$ with $\lambda \geq \lambda_{0}$.

We can therefore assert the following existence result.

Theorem 9. Let $m$ satisfy (2) and (3) and let $\phi \in L^{2}(\partial Q)$. Then there exists a unique solution in $H_{l o c}^{1,2}(m, m)$ of the problem $(1 m, \lambda),(22)$ for $\lambda \geq \lambda_{0}$.

This follows by a straightforward approximation argument (for details see the proof of Theorem 6 in [2]).

\section{References}

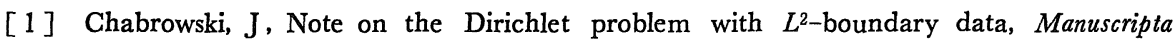
Math. 40 (1982), 91-108.

[2] Chabrowski, J., and H. B. Thompson, On the boundary values of the solutions of linear elliptic equations, Bull. Austral. Math. Soc. 27 (1) (1983), 1-30.

[3] Gilberg, D., and N.S. Trudinger, Elliptic partial differential equations of second order, Springer Verlag, Berlin-Heidelberg-New York, 1977.

[4] Mikhailov, V.P., Boundary values of the solutions of elliptic equations in domains with a smooth boundary, Mat. Sb. 101 (143) (1976), 163-188.

[5] Murthy, M. R. V., and G. Stampacchia, Boundary value problems for some degenerateelliptic operators, Ann. Mat. Pura Appl. 80 (1968), 1-122.

[6] Trudinger, N.S., Linear elliptic operator with measurable coefficients, Ann. Scuola Norm. Sup. Pisa 27 (1973), 265-308. 